\title{
Thermal Modification of bottomonium spectral functions from QCD sum rules
}

\section{Makoto Oka*}

H-27, Department of Physics, Tokyo Institute of Technology, Meguro, Tokyo 152-8551, Japan

\section{Kei Suzuki}

H-27, Department of Physics, Tokyo Institute of Technology, Meguro, Tokyo 152-8551, Japan

\section{Kenji Morita}

Yukawa Institute for Theoretical Physics, Kyoto University, Kyoto 606-8502, Japan

\section{Philipp Gubler}

RIKEN Nishina Center, Hirosawa 2-1, Wako, Saitama, 351-0198, Japan

\begin{abstract}
Quarkonium spectral functions at finite temperature are studied in QCD sum rules. We apply a recently developed method of analyzing QCD sum rules by the maximum entropy method. This approach enables us to obtain the spectral function directly from the sum rules, without having to introduce any specific assumption, such as pole plus continuum, about its functional form. Finite temperature effects are manifested in the gluon condensates that appear in the operator product expansion. We extract their temperature dependences from quenched lattice QCD calculations. It is found that the charmonium ground states of both S-wave and P-wave channels dissolve into the continuum already at temperatures around or slightly above the critical temperature $T_{c}$, while the bottomonium states are less influenced by temperature effects, surviving up to about $2.5 T_{c}$ or higher for S-wave and about 2.0 $T_{c}$ for P-wave states. Our results are consistent with recently observed LHC data, which suggest that the excited bottomonia dissociate at lower temperature than the ground state.
\end{abstract}

The 7th International Workshop on Chiral Dynamics,

August 6 -10, 2012

Jefferson Lab, Newport News, Virginia, USA

\footnotetext{
*speaker (E-mail: oka@th.phys.titech.ac.jp)
} 


\section{Introduction}

Chiral symmetry is one of the most important properties of hadron dynamics and its origin in the underlining theory, the quantum chromodynamics (QCD), is now understood fairly well. This symmetry is spontaneously broken in the vacuum of QCD and determines the overall features of meson spectrum and hadron interactions. There is, however, an interesting question not yet answered, that is, the behavior of the QCD vacuum at finite temperature and density, where chiral symmetry is expected to be restored. Similarly, the color confinement is supposed to disappear, resulting quark-gluon gas phases at high temperature and/or density.

In extracting phase information of high temperature matter of quarks and gluons, it is useful to study properties of hadrons in such matter. In particular, bound states of a quark and an antiquark, quarkonia, have often been used as probe of the matter, as in such systems both perturbative and non- perturbative interactions of QCD play important roles. It was, for instance, suggested [W] that the J/ $\psi(c \bar{c})$ meson will melt due to the color Debye screening in the color-deconfined medium and thus serve as a signal for formation of quark-gluon plasma. It was recently reported that bottomonium $(b \bar{b})$ spectra have been modified significantly in the $\mathrm{Pb}-\mathrm{Pb}$ collisions at the Large Hadron Collider (LHC) [■, []].

In these proceedings, we investigate the quarkonium spectra from a theoretical point of view, using a novel approach [䧃], based on QCD sum rules [5] and the maximum entropy method (MEM). QCD sum rules provide a powerful tool for investigating the properties of hadrons at finite temperature directly from QCD [國. The usual strategy of analyzing the sum rules is to make some reasonable assumptions on the functional form of the spectral function, and then extract information on the lowest lying peak from the results of the operator product expansion (OPE). This method, however, has several shortcomings. First of all, the widely used "pole + continuum" ansatz often works well at $T=0$, but may not be appropriate at temperatures above $T_{c}$. Furthermore, it is not always possible to unambiguously fit a specific ansatz to the OPE-results, because of the occurrence of equally valid solutions. To handle these problems, we propose to use MEM, which allows us to extract the spectral function from the OPE without prejudice on its functional form. In these proceedings, we can only show the most important results of our investigation, while more details can be found in [प, []].

\section{Formalism}

The QCD sum rule approach makes use of the analytic properties of the two-point correlation function of a local operator, $J(x)$, which in this study stands for

$$
\bar{h} \gamma^{\mu} h(x), \quad \bar{h} \gamma^{5} h(x), \quad \bar{h} h(x), \quad\left(q^{\mu} q^{v} / q^{2}-g^{\mu v}\right) \bar{h} \gamma^{5} \gamma_{v} h(x)
$$

in the vector, pseudoscalar, scalar and axial- vector channel, respectively. $h(x)$ here represents either a charm- or bottom-quark field. Using a dispersion relation, one can connect the values of the correlator calculated in the deep-Euclidean region to an integral of the spectral function $\rho(s)$. After applying the Borel transform, one arrives at the following expression:

$$
G\left(M^{2}\right)=\int d s e^{-s / M^{2}} \rho(s) d s
$$


In this equation, the left-hand side can be calculated analytically using the operator product expansion (OPE). To obtain the spectral function $\rho(s)$, one therefore has to invert the integral of Eq. (‥ لل This is, however, an ill-posed problem and can not be solved rigorously. Nevertheless, using Bayes' theorem, it is possible to obtain the the most probable form of $\rho(s)$, given Eq. (Lلd) and additional information on the spectral function such as positivity and asymptotic values. This is the essential idea of the MEM approach, for the concrete implementation in the case of QCD sum rules, see [四].

For quarkonia at temperatures not too much above $T_{c}$, all the finite temperature effects can be included into the temperature dependent condensates owing to the large separation scale in the OPE

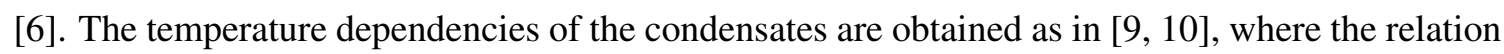
between the gluon condensate and the energy momentum tensor is employed to give the value of the gluon condensate as a function of the energy density and pressure. These thermodynamic quantities are then extracted from quenched lattice QCD calculations.

\section{Results}

The results of the charmonium and bottomonium spectral functions at zero and finite temperatures are shown in Fig. $\square$ and Fig. \. In the case of charmonium, all the peaks are suppressed at a temperature around or slightly above the critical temperature $T_{c}$. On the other hand, the bottomonium peaks survive at higher temperatures than the charmonium peaks. This difference of the melting temperatures is caused by the Wilson coefficients of the gluon condensate terms which are inversely proportional to the fourth power of the heavy quark mass.

The positions of the lowest peaks, given in Table W, are consistent with the observed masses of the ground states in the case of the charmonia, while for the bottomonia, all the peaks at $T=0$ are found $150-500 \mathrm{MeV}$ above the values of the ground state. This shift is caused by the presence of the excited states, which, in contrast to the charmonium case, is relatively large contribution to the lowest peak.

To investigate thermal behavior of the excited states, we analyze the residue of the obtained peak for the vector channel. In order to exclude the continuum contributions to the peak and to estimate the sum of the residues only of the ground and excited states, we fit the obtained spectral functions by using a Breit-Wigner function for the peak and some suitable parametrization for the continuum. The results are shown in Fig. [3. It is observed that the residue of the $\Upsilon$ peak decreases gradually with increasing temperature and approaches a constant value at higher temperature. Especially, a rapid reduction of the residue is seen at $T / T_{c}=1.5-2.0$. If we assume the disappearance of the excited states at lower temperature than the ground state, our results suggest that $\Upsilon(2 S)$ and $\Upsilon(3 S)$ disappear at $T / T_{c}=1.5-2.0$, while $\Upsilon(1 S)$ survives up to $T / T_{c}=3.0$.

\begin{tabular}{|c||c|c|c|c||c|c|c|c|}
\hline & $m_{J / \psi}$ & $m_{\eta_{c}}$ & $m_{\chi_{c 0}}$ & $m_{\chi_{c 1}}$ & $m_{\Upsilon}$ & $m_{\eta_{b}}$ & $m_{\chi_{b 0}}$ & $m_{\chi_{b 1}}$ \\
\hline Results $[\mathrm{GeV}]$ & 3.06 & 3.02 & 3.36 & 3.50 & 9.56 & 9.51 & 10.15 & 10.42 \\
\hline Exp. $[\mathrm{GeV}]$ & 3.097 & 2.980 & 3.414 & 3.510 & 9.460 & 9.389 & 9.859 & 9.893 \\
\hline
\end{tabular}

Table 1: Peak positions of the spectral functions at zero temperature from MEM and the experimental values for the quarkonium ground states. 

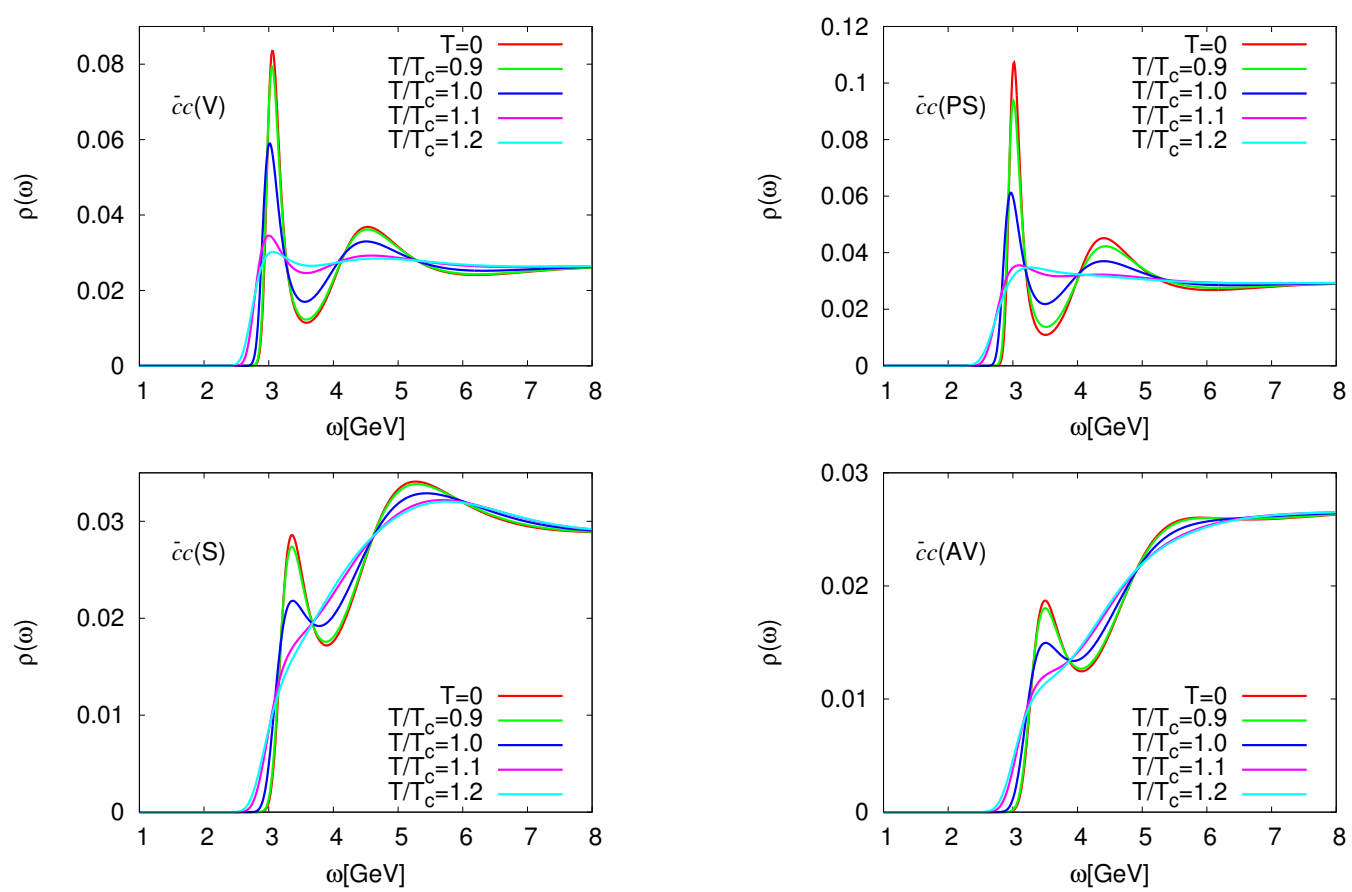

Figure 1: Spectral functions of charmonia at finite temperature from MEM. Upper left : vector $(J / \psi)$, upper right : pseudoscalar $\left(\eta_{c}\right)$, lower left : scalar $\left(\chi_{c 0}\right)$, lower right : axial-vector $\left(\chi_{c 1}\right)[\mathbb{Z}]$.
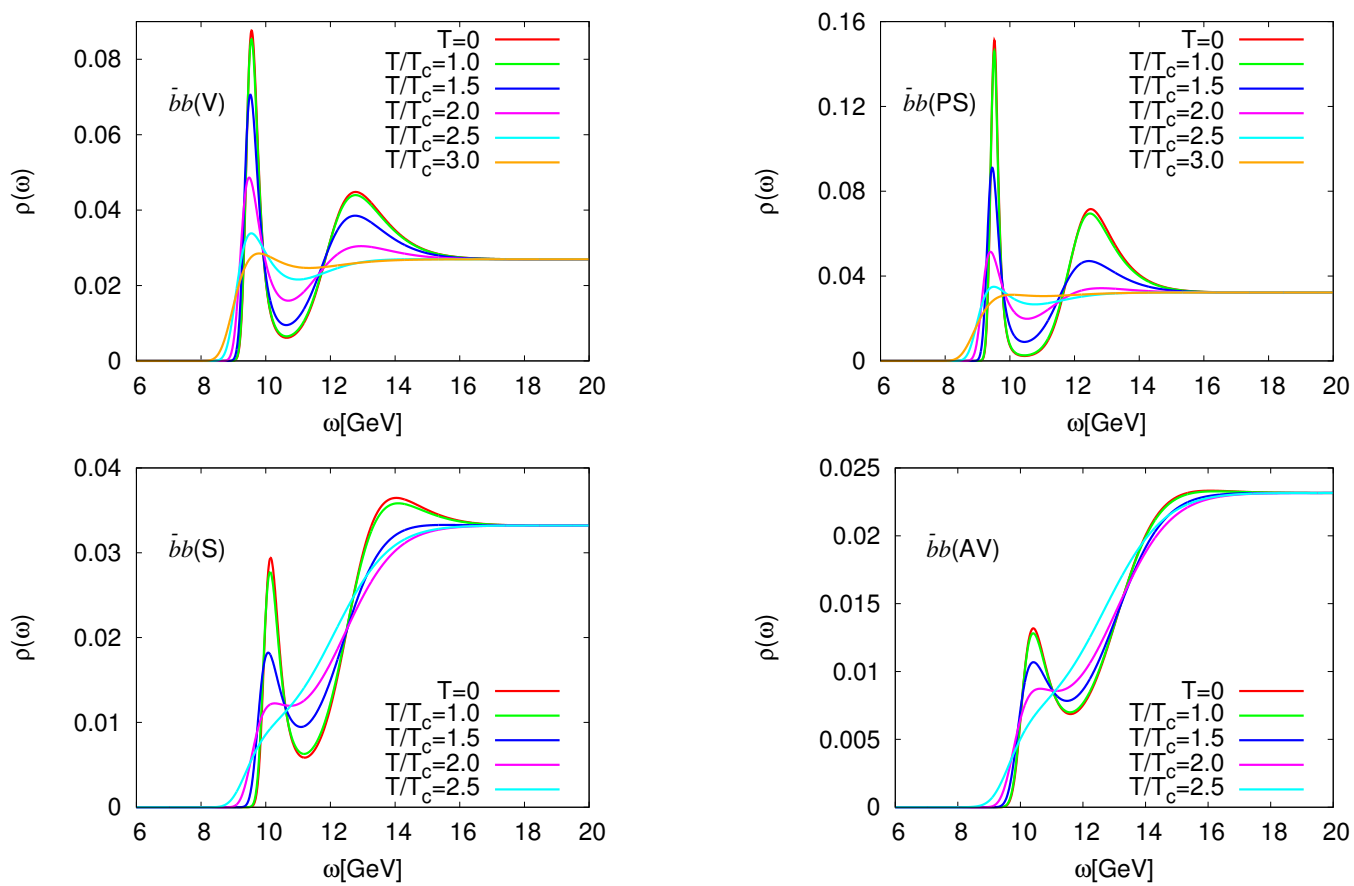

Figure 2: Spectral functions of bottomonia at finite temperature from MEM. Upper left : vector $(\Upsilon)$, upper right : pseudoscalar $\left(\eta_{b}\right)$, lower left : scalar $\left(\chi_{b 0}\right)$, lower right : axial-vector $\left(\chi_{b 1}\right)$ [ $[\mathbf{l}]$. 


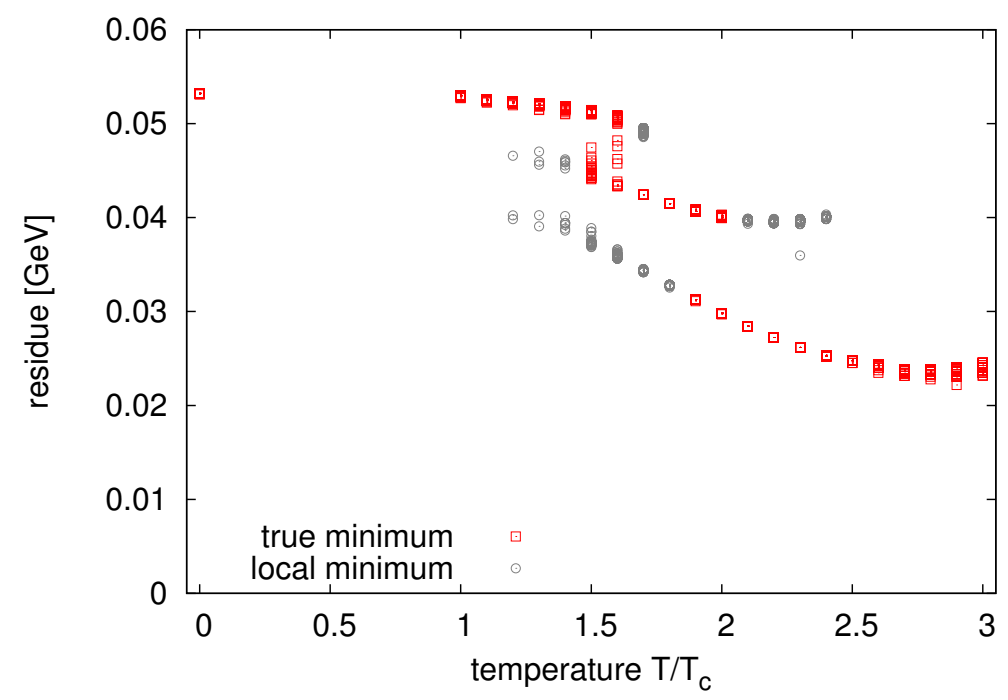

Figure 3: Temperature dependence of the residue for the vector channel bottomonium peak fitted with a Breit-Wigner + continuum. Squares are corresponding to true minima, and circles are local minima [8].

\section{Conclusion}

The quarkonium $(c \bar{c}$ and $b \bar{b})$ spectral functions at zero and finite temperatures have been analyzed in the QCD sum rules and MEM. We have applied a novel method of analyzing QCD sum rules by the maximum entropy method, which enables us to obtain the spectral function directly from the sum rules, without assuming the pole plus continuum form. Finite temperature effects are taken into account in the gluon condensates that appear in the operator product expansion. We extract their temperature dependences from quenched lattice QCD calculations.

We have found that the charmonia states, $J / \Psi, \eta_{c}$, and $\chi_{J}$, disappear at temperatures around or slightly above $T_{c}$, while the bottomonia states stay further at higher temperatures. Namely, the Swave $(\Upsilon)$ states survive up to about $2.5 T_{c}$ or higher and the P-wave states $\left(\chi_{b J}\right)$ dissolve at around $2.0 T_{c}$. From the temperature dependence of the residue of the lowest peak, we further extract information on the excited $\Upsilon$ states and have observed that the residue decreases with increasing temperature. The results are consistent with the experimental behaviors observed at LHC [Z, []].

\section{References}

[1] T. Matsui and H. Satz, Phys. Lett. B 178, 416 (1986).

[2] S. Chatrchyan et al. [CMS Collaboration], Phys. Rev. Lett. 107, 052302 (2011) [arXiv:1105.4894 [nucl-ex]]

[3] S. Chatrchyan et al. [CMS Collaboration], Phys. Rev. Lett. 109, 222301 (2012) [arXiv:1208.2826 [nucl-ex]]

[4] P. Gubler and M. Oka, Prog. Theor. Phys. 124, 995 (2010) [arXiv:1005.2459 [hep-ph]].

[5] M.A. Shifman, A.I. Vainshtein, and V.I. Zakharov, Nucl. Phys. B147, 385 (1979); B147, 448 (1979). 
[6] T. Hatsuda, Y. Koike and S. Lee, Nucl. Phys. B394, 121 (1993).

[7] P. Gubler, K. Morita and M. Oka, Phys. Rev. Lett. 107, 092003 (2011) [arXiv:1104.4436[hep-ph]].

[8] K. Suzuki, P. Gubler, K. Morita and M. Oka, Nucl. Phys. A897, 28 (2013) [arXiv:1204.1173 [hep-ph]].

[9] K. Morita and S. H. Lee, Phys. Rev. Lett. 100, 022301 (2008) [arXiv:0704.2021 [nucl-th]].

[10] K. Morita and S. H. Lee, Phys. Rev. D 82, 054008 (2010) [arXiv:0908.2856 [hep-ph]]. 